\title{
Properties of Anisotropic Interaction of the Incommensurate Superstructure as Described by Dziloshinsky’s Invariant
}

\author{
S. Sveleba, I. Katerynchuk, I. Kunyo, I. Karpa \\ Ivan Franko National University of Lviv \\ 107 Tarnavsky St., \\ UA-79017 Lviv, Ukraine \\ e-mail: incomlviv@gmail.com
}

\begin{abstract}
BDF method was applied for calculation of phase portrait of incommensurate superstructure with the Dziloshinsky's invariant. The Python language was used to create the correspond application. The value of the parameter of the anisotropic interaction is determined by the number of existing harmonics of the IC of the modulation wave. The contribution of anisotropic interaction in the dynamics of the IC superstructure grows if numbers of harmonics of the IC modulation are growing. According to the received attractors, this system is less stable at lower values of $n$.
\end{abstract}

Index Terms-anisotropic interaction; BDF method; Python language; incommensurate superstructure; Dziloshinsky's invariant; phase system portrait.

One of the peculiarities of the existence of incommensurate superstructures is the localization of the wave vector of incommensurability at comparable values of higher order. The nature of this phenomenon is pinning (fixing) the wave of incommensurate in defects and impurities [1]. Under these conditions, the magnitude of the incommensurate vector is a rational number, which can be written as a ratio of two integer $m / n$, where $m$ and $n$ are integer positive numbers. It should be noted that the number $n$ characterizes the symmetry of the thermodynamic potential, and determines the magnitude of the anisotropic interaction, which is described by the Dzyaloshinsky invariant [2] (1). In dimensionless variables $\eta=(r /(2 u))^{1 / 2} R, z=(\gamma / r)^{1 / 2} \xi$, the free energy functional for a two-component order parameter in polar coordinates has the form:

$$
\begin{gathered}
\Phi=\int \frac{r^{2}}{2 u}\left(-R^{2}+\frac{R^{4}}{2}+\frac{\omega r^{\frac{n}{2}-2}}{(2 u)^{\frac{n}{2}-1}} R^{n}(1+\cos n \varphi)-\frac{\sigma}{r^{\frac{1}{2}} \gamma^{\frac{1}{2}}} R^{2} \varphi^{\prime}+\left(R^{\prime}\right)^{2}+R^{2}\left(\varphi^{\prime}\right)^{2}\right)+ \\
\int d \xi \frac{r^{2}}{2 u}\left\{\frac{\beta r}{\gamma}\left[\left(R^{\prime \prime}-R \varphi^{\prime 2}\right)^{2}+\left(2 R^{\prime} \varphi^{\prime}+R \varphi^{\prime \prime}\right)^{2}\right]\right\}-\frac{\alpha r}{4 u} R^{2}
\end{gathered}
$$

The variation of the free energy functional (1) gives a system of two equations for phase and amplitude functions:

$$
R^{\prime \prime}-R^{3}+\left(1-\varphi^{\prime 2}+T \varphi^{\prime}\right) R-R^{n-1} K(\cos n \varphi+1)=0,
$$

$$
\varphi^{\prime \prime}+2 \frac{R^{\prime}}{R}\left(\varphi^{\prime}-\frac{T}{2}\right)+R^{n-2} K \sin n \varphi=0
$$$$
\text { here: } \quad T=\frac{\sigma}{(\gamma r)^{\frac{1}{2}}}, K=2^{-\frac{n}{2}} r^{\frac{n}{2}-2} n \omega u^{1-\frac{n}{2}}
$$

- dimensionless parameters, $n$ is an integer characterizing the potential symmetry.

Thus, according to expression (2), the parameter $K$, which characterizes the anisotropic interaction, depends on the value of $n$. Assume that the coefficient of expansion of the thermodynamic potential is less than one, then with increasing $n$ the parameter $K$ will nonlinearly decrease. This finds a good correlation with the experimental studies of the metastabile states of the incommensurate superstructure. Namely, the higher the value of $n$ causes the lower temperature interval of the existence of metastabile states.

Consequently, the study of the influence of the symmetry of the temodynamic potential on anisotropic interaction, described by the Dzyaloshinsky's invariant was carried out. Namely, the study of phase portraits of this system was obtained. The phase portrait is a complete set of different phase trajectories. He clearly illustrates the behavior of the system and its basic properties, such as equilibrium points. With the help of phase portraits you can analyze the state of stability and the nature of the movements of the system.

The construction of phase portraits of a nonlinear dynamical system is carried out in the Python software environment using the scipy library. In this library, the class scipy.integrate.ode ( $\mathrm{f}$, jac $=$ None) is the common interface class to numeric integrators. This class solves the system of equations $\left(\left(y^{\prime}(t)=f(t, y)\right)\right.$ with jac $=\mathrm{d} f / \mathrm{d} y$. Using the set integrator method, the integrator "vode", which is the usual solver of the differential equation, with the introduction of a fixed-leading coefficient. The leading factor is the integer in the set integrator class of the ode class, which takes the following parameters [3]: 
atol: floating or definite accuracy (in this work it was chosen equal to atol $=1 \mathrm{E}-6 \div-12$ )

rtol: relative permissible parameter $(\mathrm{rtol}=0)$;

method: " adams " or 'bdf'. In the calculation, the solver was used as "Adams" (for non-hard systems) and "BDF" (for hard systems);

with_jacobian: bool this parameter was entered when the Jacobian function was not considered and did not indicate that the Jacobian was grouped.

Figure 1 shows the program code. In this case, the stability parameter of the incommensurate superstructure $T=\mathrm{c} 0$, the parameter of anisotropic interaction $K=\mathrm{c} 1$, and $n=\mathrm{c} 2$. The number of calculated points was selected $200 \div 10,000$ in increments of 0.0004 (Fig. 1).

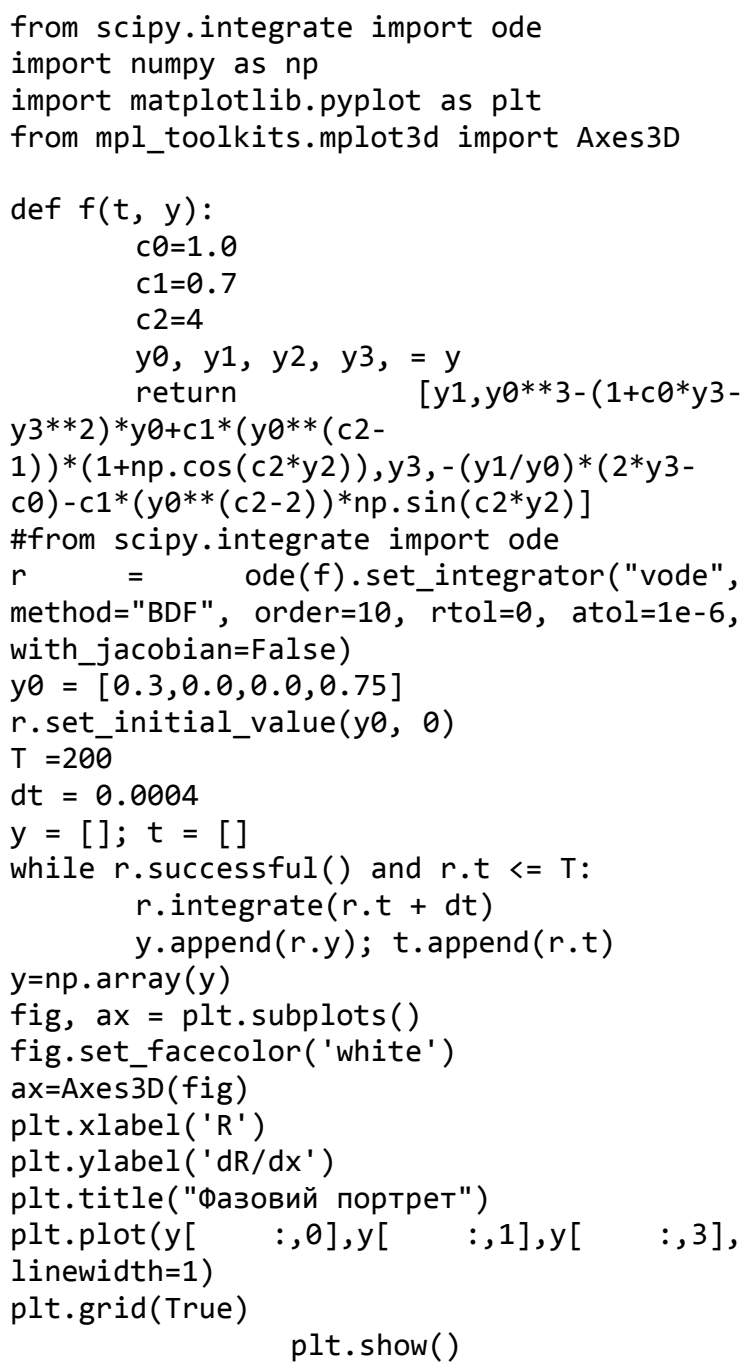

Figure 1. Application code.

Fig. 2 shows phase portraits with constant values of $K(K=$ 1.36) and $T(T=1.0)$ with a variable $n$. Paired values of $n$ characterize the ferroelectric nature of the incommensurate superstructure, and is odd-ferroelectric nature of its nature. That is, at paired values of $n$, the secondary order parameter is spontaneous polarization which is spatially modulated in the IC phase. Provided that when $n$ is an odd number, the secondary parameter of order is spontaneous deformation [4]. For both paired and odd values of $n$, if its value increases, a decrease in the number of bifurcations and a transition to an attractor which takes the form of a plume type can be traced. Such an attractor is characteristic of incommensurate superstructures which are in sinusoidal mode of modulation. The character of this attractor is particularly well manifested when the number of calculated points increases $(1000 \div 100000)$.

Thus, an increase in the value $n$ leads to a decrease in the value of the parameter of anisotropic interaction $K$. This conclusion correlates well with experimental studies of the value of the temperature interval of the existence of longperiodic proportional phases from the value of $n$ [4].
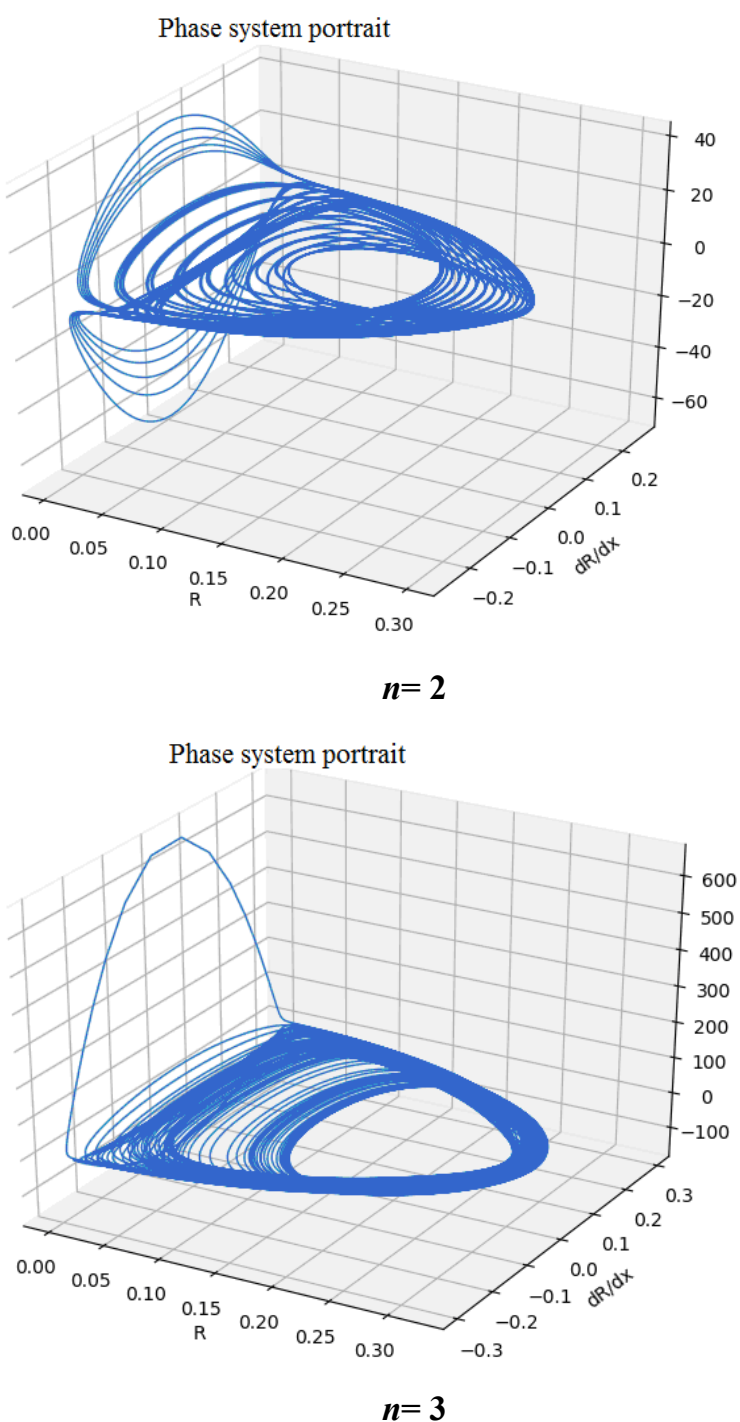

$n=3$ 


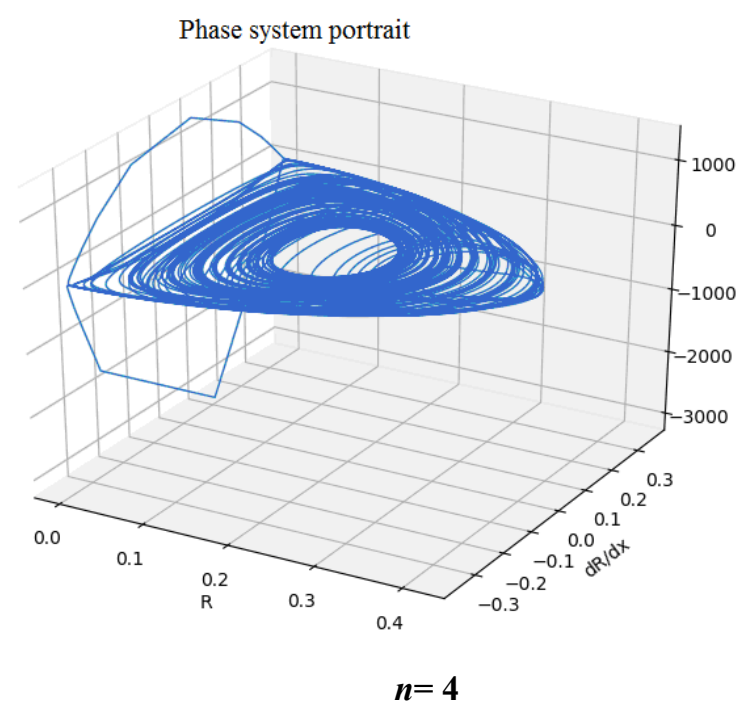

It is known [5] that an incommensurate superstructure is characterized by a sinusoidal and a soliton modulation regime. In a soliton regime, the superstructure is characterized by pinning on defects and impurities, resulting in the localization of the wave vector of incommensurability at commensurate of higher order values $(q=m / n)$. This testifies to the appearance of anisotropic interaction of inequality, which is described by the Dzyaloshinsky invariant. With the subsequent increase in the number of harmonics there is a process of increasing anisotropic interaction leading to the emergence of a stochastic regimen of IC modulation with the onset of a chaotic phase [5].
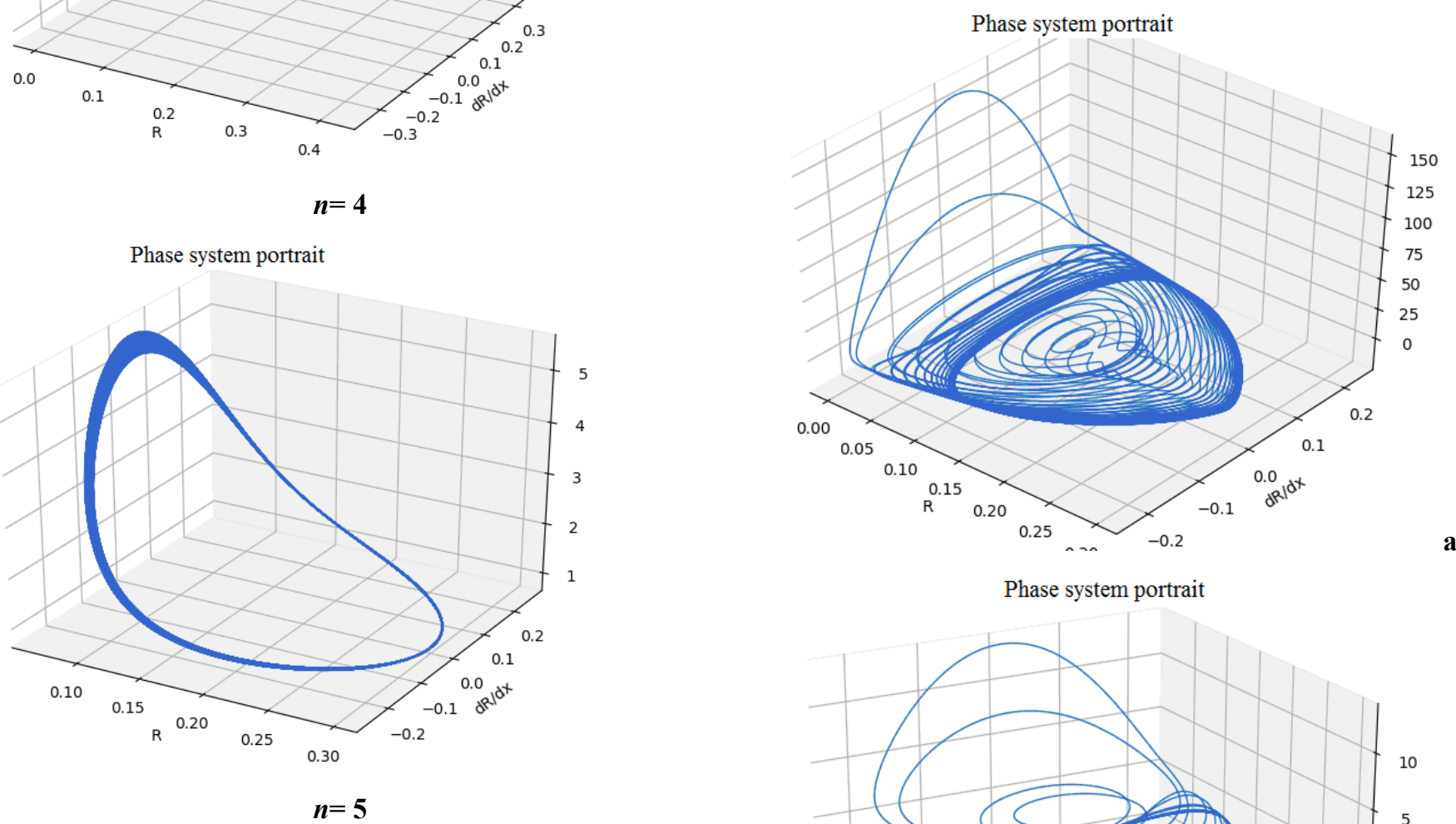

Phase system portrait
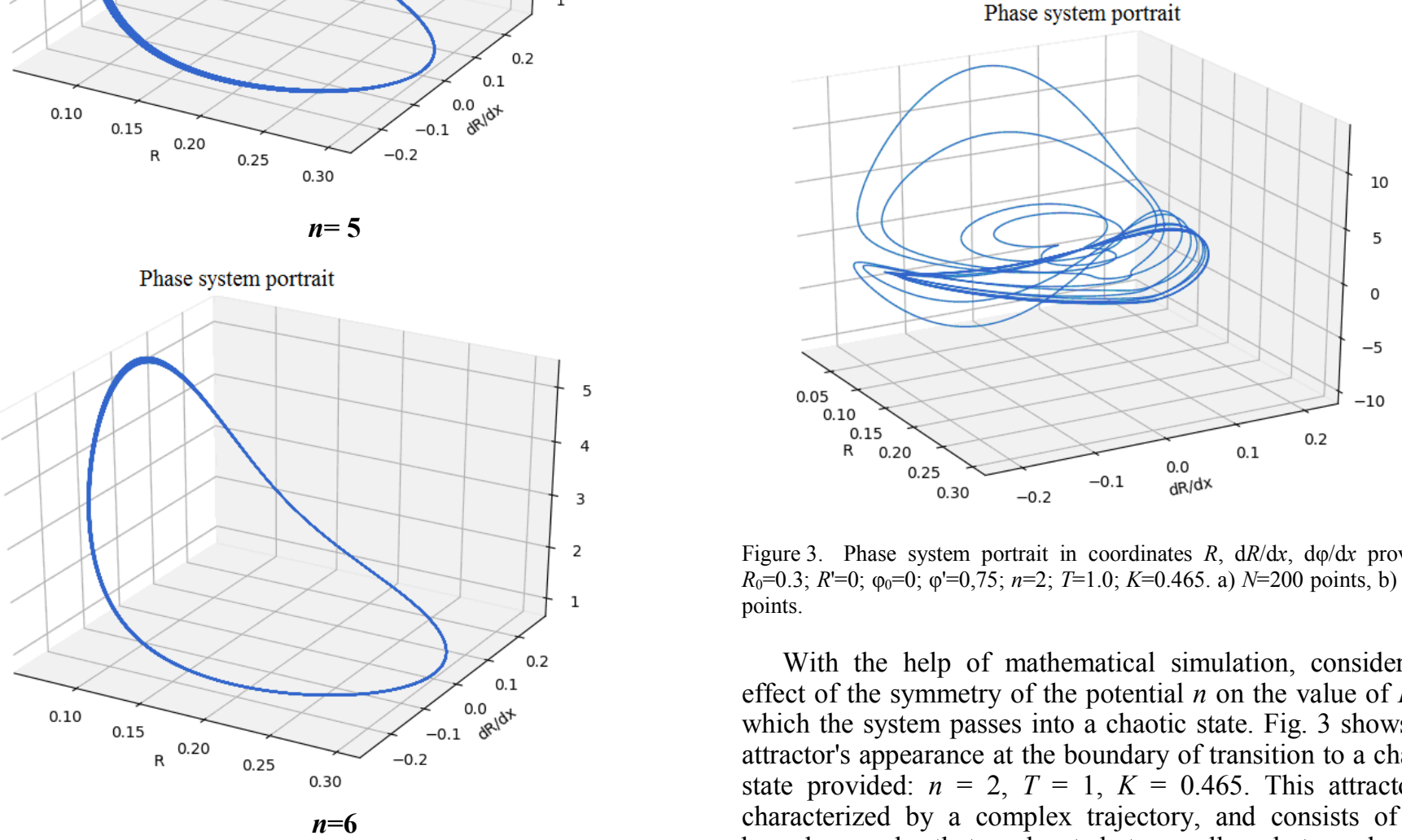

b

Figure 3. Phase system portrait in coordinates $R, \mathrm{~d} R / \mathrm{d} x, \mathrm{~d} \varphi / \mathrm{d} x$ provided: $R_{0}=0.3 ; R^{\prime}=0 ; \varphi_{0}=0 ; \varphi^{\prime}=0,75 ; n=2 ; T=1.0 ; K=0.465$. a) $N=200$ points, b) $N=50$ points.

With the help of mathematical simulation, consider the effect of the symmetry of the potential $n$ on the value of $K$, in which the system passes into a chaotic state. Fig. 3 shows the attractor's appearance at the boundary of transition to a chaotic state provided: $n=2, T=1, K=0.465$. This attractor is characterized by a complex trajectory, and consists of two boundary cycles that are located at a small angle to each other. With an increase in the value of $n$ the character of the attractor changes on the verge of transition to a chaotic state. So, the value of the parameter $K=214.5$ (Fig. 4). In other words,

Figure 2. Phase system portrait in coordinates $R, \mathrm{~d} R / \mathrm{d} x, \mathrm{~d} \varphi / \mathrm{d} x$ provided: $R_{0}=0.3 ; R^{\prime}=0 ; \varphi_{0}=0 ; \varphi^{\prime}=0.75 ; T=1 ; K=1,36$ at different values of $n$. 
taking into account the results of work [6], the transition to chaos at large values of $n(n>5)$ occurs as a result of bifurcation, and at lower values $n$ the transition to chaos is carried out through the intermediate chaotic phase.
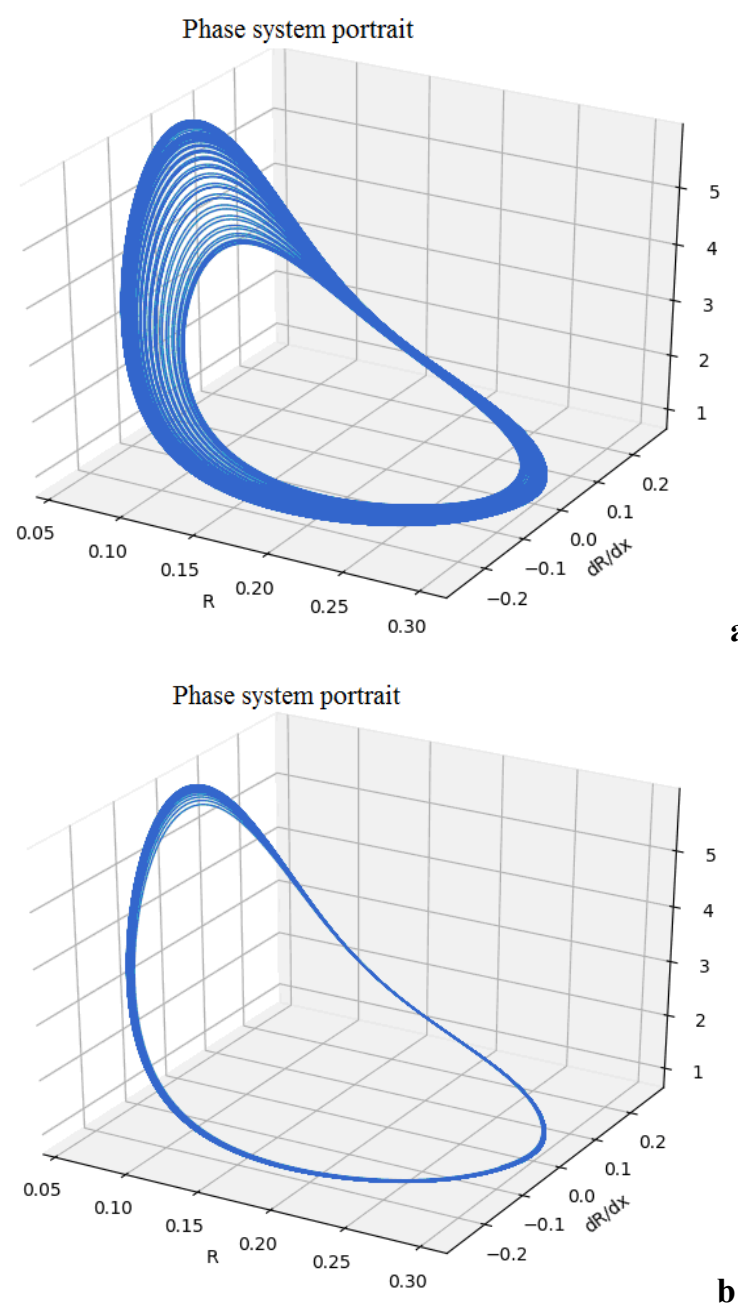

Figure 4. Phase system portrait in coordinates $R, \mathrm{~d} R / \mathrm{d} x, \mathrm{~d} \varphi / \mathrm{d} x$ provided: $R_{0}=0.3 ; R^{\prime}=0 ; \varphi_{0}=0 ; \varphi^{\prime}=0,75 ; n=8 ; T=1.0 ; K=214.5$. a) $N=200$ points, b) $N=50$ points.

Carrying out similar calculations for various $n$, it can be argued that the dependence $K \sim f(n)$ is a nonlinear and power function. To explain this dependence we consider the parameter of anisotropic interaction in the form: $K=(r / 2 u)^{n / 2} n\left(\omega u / r^{2}\right)$. Assuming that the coefficients of the decomposition of the thermodynamic potential are small variables, then this expression can be rewritten in the form $K \sim a^{\mathrm{n} / 2} n b$, where $a=r / 2 u, b=\omega u / r^{2}$. Comparing the dependence $K \sim f(n)$ obtained experimentally and theoretically calculated, it should be noted that in the first approximation there is a good correlation between them.

Thus, the anisotropic interaction described by the Dzyaloshinsky's invariant depends on the symmetry of the thermodynamic potential. As the magnitude $n$ increases, the parameter $T$ is nonlinear (according to the power law) decreases.

Proceeding from the results shown in Fig. 3 and Fig. 4, the value of the parameter of the anisotropic interaction is determined by the number of existing harmonics of the IC of the modulation wave. The contribution of anisotropic interaction in the dynamics of the IC superstructure grows if numbers of harmonics of the IC modulation are growing. According to the received attractors, this system is less stable at lower values $\mathrm{f} n$.

Hence, a richer picture of the dynamics of the IC superstructure should be expected at lower values of $n$. Under this condition, the parameter of anisotropic interaction becomes proportional (we may say identically) of the stability of the IC superstructure (parameter $T$ ).

Regarding the nature of the modulation of the secondary order parameter (ferroelectric or ferroelastic), it does not affect the character of the anisotropic interaction of the IC modulation.

\section{REFERENCES}

[1] Cummins H.Z. Experimental Studies of structurally incommensurate crystal phases Physics Reports. - 1990. - Vol.185, N 5,6. - P. 211-409.

[2] Izyumov Yu.A., Syromyatnikova V.M. Phase transitions and crystal symmetry. - Moscow: Nauka, 1984. - 248 p. [in Russian]

[3] https://docs.scipy.org/doc/scipy/reference/generated/scipy.integrate.ode. html

[4] Sveleba Sergey Andreevich. Spatially-modulated states in dielectric crystals with an incommensurate phase: dis ... Dr. phys.-math. Sciences: 01.04.07 Lviv National University. Ivan Franko - L., 2006. - 393 ark. bibliographer: arch. 353-393. [in Ukrainian]

[5] Bak P. Chaotic Behavior and Incommensurate Phases in the Anisotropic Ising Model with Competing Interactions Phys. Rev. Vol. Lett. - 1981. Vol. 46. - P. 791-794.

[6] Kuznetsov, SP Dynamic Chaos / - Moscow: Physcmatlit, 2001. - 296 pp. [in Russian] 